\title{
Avaliação do Potencial da Spirulina Maxima na Remoção da Cor em Efluentes Oriundos do Beneficiamento Têxtil
}

\author{
Vanessa T. Pereira, Ronaldo L. de Souza, Flávia D. Pimenta \& Marta C. Picardo
}

O trabalho estudou a cinética de biossorção do corante Acid Blue 324 pela cianobactéria Spirulina maxima. Avaliou-se a capacidade de remoção da cor do referido corante, o qual foi adicionado à cultura da cianobactéria Spirulina maxima. Foram avaliados dois fatores, a concentração de nitrato e a concentração de corante. O estudo demonstrou bons resultados na remoção do corante do meio de cultura com redução expressiva da cor, alcançando valores acima de $80 \%$ na remoção da cor na cultura.

Palavras-chave: acid blue 324; Spirulina maxima; biossorção.

The work studied the biosorption kinetics of the dye Acid Blue 324 by the cyanobacteria Spirulina maxima. It was evaluated the color removal capacity of the said Dye, which was added to the culture of the cyanobacteria Spirulina maxima. Two factors were evaluated, the concentration of nitrate and the concentration of dye. The study demonstrated good results in the removal of the dye from the culture medium with expressive reduction of the color, reaching values above $80 \%$ in the removal of the color in the culture.

Keywords: acid blue 324; Spirulina maxima; biosorption. 


\section{Introdução}

A indústria têxtil possui um dos processos que mais geram poluentes para o meio ambiente, em vista do descarte de corantes em efluentes não tratados corretamente ${ }^{1}$, dos 100.000 tipos usados nos processos de tingimento, cerca de 10 a 50\% destes são perdidos nos efluentes industriais ${ }^{2}$.

Em geral, os corantes possuem em sua composição química, grandes quantidades de anéis aromáticos, ligações azo, aminas e grupos sulfônicos, que são reconhecidamente recalcitrantes, por terem um lento processo de biodegradação e elevados potenciais toxicológico 3 . Uma vez dispersos em corpos d'água, eles reduzem a transparência da água afetando a atividade fotossintética e a concentração de oxigênio dissolvido essencial para a vida aquática ${ }^{4}$.

As principais técnicas disponíveis na literatura para a descoloração das águas de rejeitos industriais envolvem principalmente os processos de adsorção, precipitação, degradação química, eletroquímica e fotoquímica, biossorção, entre outros ${ }^{5}$. A biossorção é um processo físico-químico complexo, geralmente, envolvendo mais de um mecanismo (por exemplo, complexação, adsorção, troca iônica, precipitação, etc.) e caracterizado por uma cinética rápida ${ }^{6}$.

Algumas biomassas podem ser utilizadas como biossorventes, tais como leveduras, bactérias, algas e fungos, adsorvendo íons metálicos de soluções contaminadas?

Diante dessa capacidade de biossorção das algas, os estudos nos últimos anos com o uso de microalgas e cianobactérias na descoloração de águas residuais têm atraído grande interesse, aliado à sua capacidade em se desenvolver rapidamente em ambientes com elevadas cargas de nitrogênio e fósforo inorgânico e na mitigação de dióxido de carbono $\left(\mathrm{CO}_{2}\right)$; além desses fatores, a biomassa gerada tem um grande potencial como matéria-prima para a produção de biocombustíveis e energia ${ }^{8-10}$.

A cianobactéria Spirulina maxima possui a capacidade para sobreviver em um ambiente extremo, devido ao modo fototrófico de nutrição, tempo de geração curto e fácil cultivo gerando elevada concentração em massa ${ }^{11}$.
Tomou-se, então, como objetivo deste trabalho, utilizar a Spirulina maxima como uma alternativa de baixo custo e de menor impacto ambiental para a remoção da cor de uma solução aquosa contendo um corante sintético utilizado no tingimento de tecidos nas indústrias têxteis.

\section{Metodologia}

Avaliação da influência da concentração de corante e da concentração de nitrato no meio de cultura no crescimento da cianobactéria Spirulina máxima

O planejamento de experimentos usado para estudar o efeito das concentrações de nitrato e de corante no crescimento celular obtido no início da fase estacionária. Realizou-se um planejamento fatorial $2 \mathrm{~K}$ com ponto central (0), m que $\mathrm{K}$ é o número de fatores e 2 o número de níveis. Esses níveis são chamados de superiores $(+)$ e de inferiores $(-)$, resultando numa condição de $22=4$ experimentos (Tabela 1) ${ }^{12,13}$. Os dados obtidos foram avaliados no software STATISTICATM'99 for Windows, versão 7.0, produzido pela StatSoft.

Tabela 1. Matriz de níveis do planejamento fatorial $2^{2}$ com ponto central.

\begin{tabular}{|c|c|c|}
\hline \multirow{2}{*}{ Experimentos } & \multicolumn{2}{|c|}{ Fatores } \\
\cline { 2 - 3 } & $\begin{array}{c}\text { X1 - } \\
\text { Concentração do } \\
\text { corante (ppm) }\end{array}$ & $\begin{array}{c}\text { X2 - } \\
\text { Concentração do } \\
\text { corante (g/L) }\end{array}$ \\
\hline 1 & $(+) 75$ & $(-1) 0,10$ \\
\hline 2 & $(-1) 25$ & $(+1) 0,50$ \\
\hline 3 & $(+1) 75$ & $(+1) 0,50$ \\
\hline 4 & $(-1) 25$ & $(-1) 0,10$ \\
\hline $5 a$ & $(0) 50$ & $(0) 0,30$ \\
\hline $5 b$ & $(0) 50$ & $(0) 0,30$ \\
\hline $5 c$ & $(0) 50$ & $(0) 0,30$ \\
\hline
\end{tabular}

\section{CONDIÇÕES OPERACIONAIS DO CULTIVO}

A Temperatura foi de $28 \pm 2{ }^{\circ} \mathrm{C}$ mantida pelo ar condicionado do laboratório; a iluminação ambiente do próprio laboratório $(384 \mathrm{~W})$ por $24 \mathrm{~h}$, isto é, sem 
fotoperíodo; agitação de $110 \mathrm{rpm}$ em placas agitadoras com agitador magnético, e um $\mathrm{pH}$ inicial (meio sem o corante) de $10,0 \pm 0,5$.

\section{MICRORGANISMO E MANUTENÇÃO DA CULTURA}

Foi usada uma cianobactéria denominada como Spirulina maxima, mantida em meio Aiba \& Ogawa ${ }^{14}$. Para manutenção da cultura, foi realizado um repique da cepa em quatro erleynmeyers de $500 \mathrm{~mL}$ (culturas estoque), com volume de $300 \mathrm{~mL}$ de meio de cultura, com $\mathrm{pH}$ de 10,0 $\pm 0,5$ e agitação de $110 \mathrm{rpm}$ em placas de agitação com agitador magnético. Foi realizado acompanhamento do crescimento celular através da espectrofotometria em um comprimento de onda igual a $680 \mathrm{~nm}$.

\section{PREPARO DO CORANTE}

O Acid Blue 324, usado no experimento, é da marca CLARITAN, de nome comercial Azul Nylosan E-BGL, seu número de registro da Chemical Abstracts Service (CAS) é 88264-80-6. Preparou-se uma solução do referido corante com uma concentração de $5 \mathrm{~g} / \mathrm{L}$ (solução estoque), sendo usado posteriormente no meio de cultura em diferentes concentrações conforme o planejamento experimental.

Após isso, foi construída uma curva padrão deste corante sendo retirado $1 \mathrm{~mL}$ da solução estoque e feitas diluições, $1: 2 ; 1: 3 ; 1: 4 ; 1: 5 ; 1: 6 ; 1: 7 ; 1: 8 ; 1: 9 ; 1: 10 ; 1: 13 ; 1: 16 ; 1: 19$; $1: 22 ; 1: 26 ; 1: 35 ; 1: 50 ; 1: 65 ; 1: 80 ; 1: 90 ; 1: 100 ; 1: 150 ; 1: 200$, baseadas na medida de absorbância da solução concentrada. Mediu-se no espectrofotômetro da marca Biochrom, modelo Libra S35, no comprimento de onda de $716 \mathrm{~nm}^{15,16}$, posteriormente determinou-se a curva padrão do corante.

\section{DETERMINAÇÃO DO NITRATO RESIDUAL}

Para o preparo da curva padrão do nitrato, foram pesados cinco valores de nitrato em gramas $(0,001 \mathrm{~g} ; 0,003 \mathrm{~g} ; 0,005$ g; $0,007 \mathrm{~g}$ e $0,010 \mathrm{~g}$ ) e diluiu-se em um volume de 1000 $\mathrm{mL}$. De cada balão volumétrico foi retirada uma alíquota e foi usado um método espectrofotométrico ${ }^{17}$, adicionouse $\mathrm{HCl} 1 \mathrm{M}$ a $(1 \% \mathrm{v} / \mathrm{v})$, e a leitura de densidade ótica foi realizada a $220 \mathrm{~nm}$.

\section{DETERMINAÇÃO DA BIOMASSA SECA}

Determinou-se a concentração de biomassa seca por volume de cultura no final do cultivo, quando as células alcançaram a fase estacionária de crescimento.

O peso seco das células foi determinado filtrando-se $200 \mathrm{~mL}$ da cultura celular em filtro de fibra de vidro com porosidade $0,45 \mu \mathrm{m}$. Os filtros utilizados foram previamente tratados em forno mufla a $540{ }^{\circ} \mathrm{C}$ por $2 \mathrm{~h}$. Antes da filtração, os filtros foram secos em estufa a $100^{\circ} \mathrm{C}$ por 2 horas, resfriados em dessecador e pesados (P1). Os filtros com biomassa foram colocados na estufa e mantidos a $40^{\circ} \mathrm{C}$ até peso constante (P2), com o objetivo de determinar o peso seco da biomassa. Os ensaios foram realizados em sextuplicata e o peso seco foi calculado como a diferença $\mathrm{P} 2-\mathrm{P} 1^{18}$.

\section{DETERMINAÇÃO DA CAPACIDADE MÁXIMA DE CAPTAÇÃO DO CORANTE PELA SPIRULINA MAXIMA}

A curva foi construída a partir da solução do corante de concentração de $75 \mathrm{mg} / \mathrm{L}$, que foi colocada em contato com a cultura de células com um volume de meio de 1000 $\mathrm{mL}$ com concentração de nitrato de $0,50 \mathrm{~g} / \mathrm{L}$. A partir do primeiro minuto, foi retirada uma alíquota de $10 \mathrm{~mL}$ do cultivo e a solução foi filtrada em filtro de fibra de vidro para separação da biomassa, todo este processo foi repetido para todas as alíquotas retiradas. Todas as soluções residuais do corante foram quantificadas usando o espectrofotômetro a $716 \mathrm{~nm}$ ao longo de 70 minutos e a água destilada foi usada como branco.

A absorbância da biomassa foi medida usando o espectrofotômetro a $680 \mathrm{~nm}$. O pH também foi monitorado e manteve-se constante em $\mathrm{pH}=10,0 \pm 0,5$.

A capacidade de biossorção foi determinada pela equação (3) abaixo $^{19}$.

$$
Q e=\frac{(C i-C f)}{x}
$$

Sendo que Qe é a quantidade de corante adsorvido por unidade de peso de biomassa (mg / g), Ci é a concentração 
inicial do corante (mg/L), Cf é a concentração final do corante após o equilíbrio $(\mathrm{mg} / \mathrm{L})$ e x é a biomassa $(\mathrm{g} / \mathrm{L})$.

\section{Resultados e Discussões}

\section{ANÁLISE ESTATÍSTICA DOS EXPERIMENTOS DE CAPTAÇÃO DO CORANTE}

A Tabela 2 abaixo mostra a matriz do planejamento experimental, com os valores das variáveis independentes (respostas). Essa matriz, para a resposta Densidade óptica (D.O.) da biomassa, possibilitou a elaboração de uma representação gráfica, que foi a superfície resposta e o cálculo dos efeitos dos fatores manipulados sobre a resposta investigada.

\section{ANÁLISE ESTATÍSTICA DA VARIÁVEL RESPOSTA DENSIDADE ÓPTICA (D.O.) DA BIOMASSA}

A matriz do planejamento apresentada na Tabela 2, para a resposta Densidade Óptica (D.O.), possibilitou a elaboração de uma representação gráfica, que é a superfície resposta e o cálculo dos efeitos dos fatores manipulados sobre a resposta investigada.

$\mathrm{O}$ efeito dos fatores manipulados sobre a resposta investigada indica a magnitude da resposta, de acordo com a variação dos níveis superiores (+) ou inferiores (-) dos fatores, como mostra a Tabela 1.

A Figura 1 a seguir apresenta o gráfico de Pareto para variável resposta D.O. Na análise do gráfico de Pareto, as barras horizontais que ultrapassaram a linha tracejada possuem efeito significativo sobre a resposta $(\mathrm{p}<0,05)$. Os valores ao lado do retângulo representam os valores da estatística do Teste t, obtidos pela tabela dos efeitos principais. Os efeitos positivos indicam que os fatores devem ser usados no nível superior $(+)$, enquanto os efeitos negativos indicam que os fatores devem ser usados no nível inferior (-), para o aumento da variável resposta.

De acordo com a Figura 1, as variáveis concentração do corante (X1), concentração de nitrato (X2), assim como a relação entre os efeitos (X1X2), não mostraram significância estatística, a um nível de 5\% de significância.

A análise do gráfico de Pareto (Figura 1) mostra que as variáveis independentes, assim como a interação entre os fatores, não apresentam para $95 \%$ de confiança, um efeito significativo sobre a resposta D.O.

A Figura 2 abaixo apresenta o gráfico da superfície resposta, para o modelo linear, da variável resposta D.O. A análise dessa Figura sugere que com a redução das concentrações do corante (abaixo de 25 ppm) e de nitrato de

Tabela 2. Matriz padrão de planejamento fatorial 22 com ponto central, tendo como variáveis resposta à Densidade óptica (D.O.) (biomassa), Concentração de corante e de Nitrato residuais.

\begin{tabular}{|c|c|c|c|c|c|}
\hline \multirow{2}{*}{ Ensaios } & \multicolumn{2}{|c|}{ FATORES } & \multicolumn{3}{c|}{ RESPOSTAS } \\
\cline { 2 - 6 } & $\begin{array}{c}\text { X1 } \\
\text { concentração de }\end{array}$ & $\begin{array}{c}\text { X2 } \\
\text { Concentração de } \\
\text { nitrato em } \\
\text { (ppm) }\end{array}$ & $\begin{array}{c}\text { Densidade Óptica } \\
\text { (Biomassa) } \\
\text { (Absorbância) }\end{array}$ & $\begin{array}{c}\text { Concentração de } \\
\text { corante residual } \\
\text { (Absorbância) }\end{array}$ & $\begin{array}{c}\text { Concentração de } \\
\text { nitrato residual } \\
\text { (Absorbância) }\end{array}$ \\
\hline 1 & $(+1) 75$ & $(-1) 0,10$ & 0,49 & 0,002 & 0,487 \\
\hline 2 & $(-1) 25$ & $(+1) 0,50$ & 0,426 & 0,013 & 0,491 \\
\hline 3 & $(+1) 75$ & $(+1) 0,50$ & 0,488 & 0,004 & 0,474 \\
\hline 4 & $(-1) 25$ & $(-1) 0,10$ & 0,417 & 0,005 & 0,494 \\
\hline $5 a$ & $(0) 50$ & $(0) 0,30$ & 0,338 & 0,017 & 0,469 \\
\hline $5 b$ & $(0) 50$ & $(0) 0,30$ & 0,421 & 0,018 & 0,473 \\
\hline $5 \mathrm{c}$ & $(0) 50$ & $(0) 0,30$ & 0,434 & 0,012 & 0,444 \\
\hline
\end{tabular}


(abaixo de 0,10 g/), verifica-se a diminuição dos valores de Densidade óptica, o que sugere menor crescimento celular em baixas concentrações de nitrato.

O crescimento das microalgas é afetado pelas condições de cultura, como a luz que é um fator indispensável para a fotossíntese, disponibilidade de nutrientes como os compostos de carbono, hidrogênio, oxigênio, nitrogênio, entre outros e também a temperatura ${ }^{20-22}$. Quando ocorrem condições estressantes no ambiente, como a falta de nutrientes e luz, as microalgas reduzem o seu crescimento, bem como a sua capacidade fotossintética, pois, a energia restante é usada para síntese de lipídios de armazenamento ${ }^{20,23,24}$.

\section{ANÁLISE ESTATÍSTICA DAS VARIÁVEIS DE RESPOSTA - CONCENTRAÇÃO DE CORANTE RESIDUAL E CONCENTRAÇÃO DE NITRATO RESIDUAL}

As Figuras 3a e 3b abaixo apresentam os gráficos de Pareto para variáveis de resposta, concentração de corante residual e nitrato residual. Assim como foi observado para a variável D.O., as variáveis manipuladas, não apresentaram efeito significativo sobre a resposta concentração de corante residual e nitrato residual.

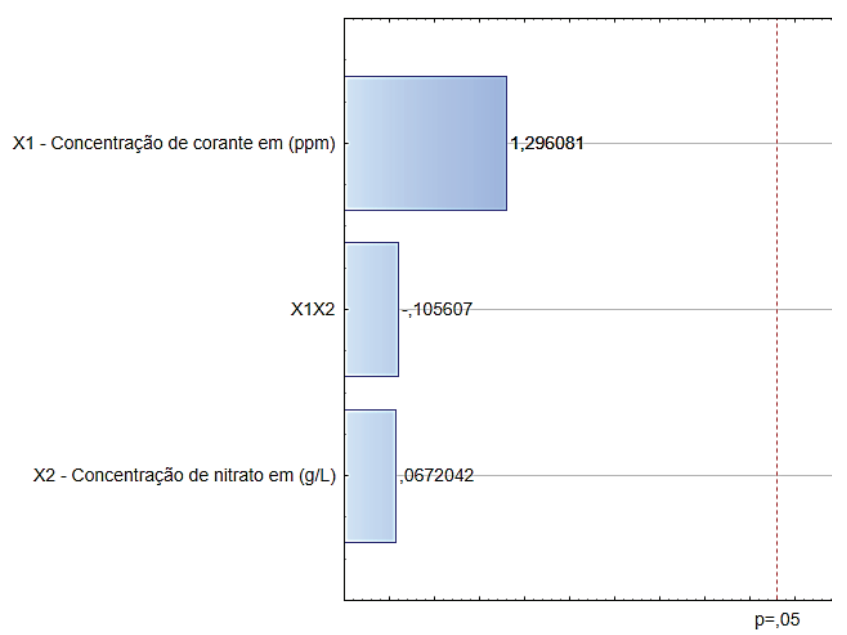

Figura 1. Gráfico de Pareto para a resposta Densidade óptica a um nível de significância de $5 \%$.
As Figuras 4a e 4b abaixo apresentam os gráficos da superfície resposta, para o modelo linear, das variáveis de resposta concentração de corante residual e nitrato residual.

A análise dos gráficos de superfície (Figuras 4a e 4b) sugere que as menores concentrações de corante residual, são obtidas a partir da redução da concentração de nitrato e do aumento da concentração de corante. Os perfis de crescimento e a análise do gráfico de superfície não apresentaram uma influência significativa dos fatores sobre as variáveis resposta para estas condições de cultivo.

A baixa luminosidade provavelmente é o parâmetro responsável pelo baixo rendimento em biomassa, isto é, nesta condição de luminosidade não há crescimento celular significativo. Dessa forma, a concentração de nitrato, mesmo em baixos níveis, não afeta o crescimento celular (D.O.), assim a luminosidade é um fator limitante e deve ser considerado no planejamento experimental.

A análise estatística permitiu verificar que não houve influência dos fatores selecionados sobre as variáveis de resposta para as condições de cultivo do presente trabalho. $\mathrm{O}$ aumento da intensidade luminosa poderia aumentar a captação do nitrato, aumentando o crescimento celular e consequentemente a captação de corante no cultivo.

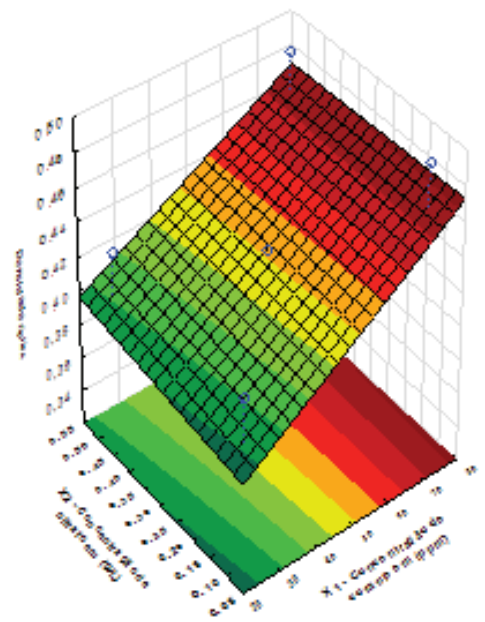

Figura 2. Superfície resposta para o modelo linear da variável Densidade Ótica. X2- concentração de nitrato em g/L, X1- Concentração de corante em ppm. 


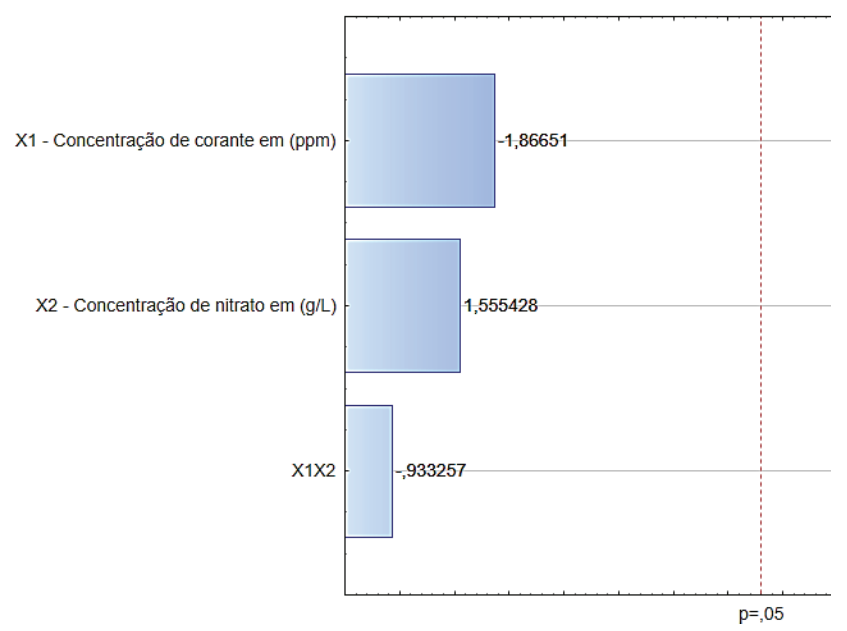

Figura 3. (a) Gráfico de Pareto para a resposta concentração de corante residual a um nível de significância de 5\%.

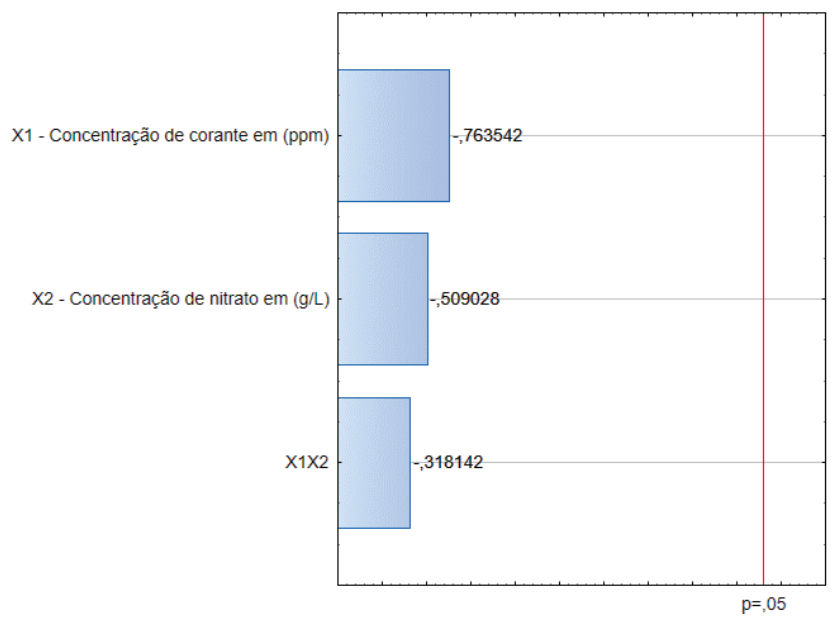

Figura 3. (b) Gráfico de Pareto para a resposta concentração de nitrato residual a um nível de significância de 5\%.

\section{AVALIAÇÃO DA CAPACIDADE DE REMOÇÃO DA COR DO CORANTE ACID BLUE 324 PELA SPIRULINA MAXIMA}

Na Figura 5 abaixo, está representada a capacidade de remoção da cor do corante Acid Blue 324 pela Spirulina maxima ao longo de 84 minutos de ensaio.

A remoção de cor ao final do ensaio alcançou máximo de 83,19 \% (captação máxima de 313,288 mg/g) em 84 minutos de biotratamento.

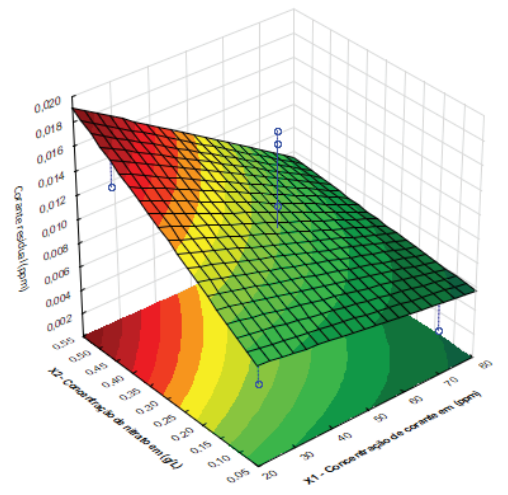

Figura 4. (a) Superfície resposta para o modelo linear da variável corante residual. X2- concentração de nitrato em g/L, X1- Concentração de corante em ppm.

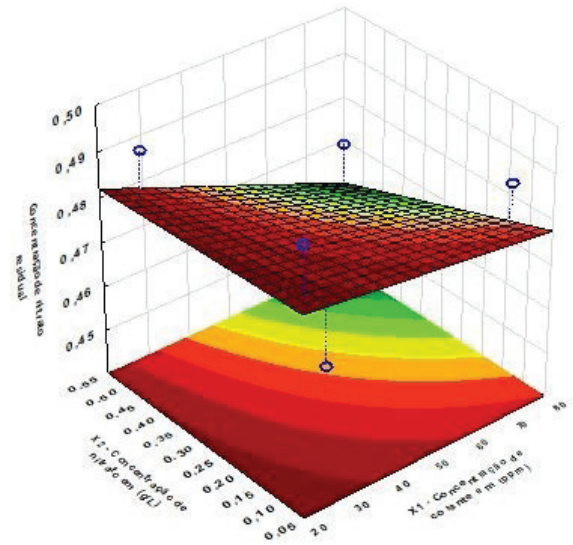

Figura 4. (a) Superticie resposta para o modelo lınear da varıavel nitrato residual. X2- concentração de nitrato em g/L, X1- Concentração de corante em ppm.

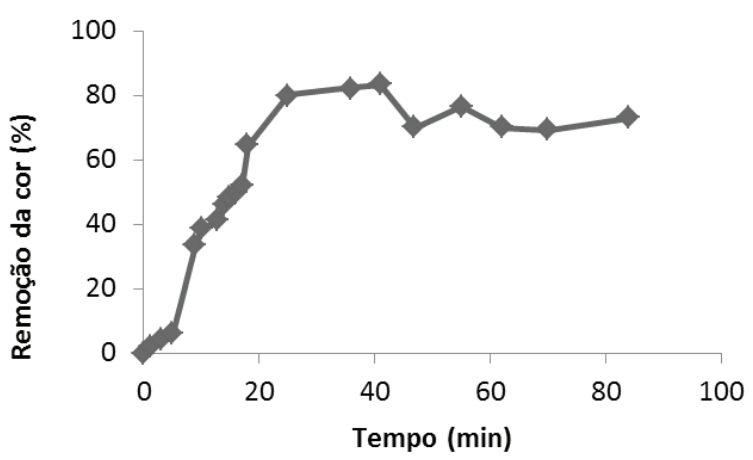

Figura 5. Porcentagem da remoção da cor do corante Acid Blue 324 durante o ensaio com a Spirulina maxima. 
Este resultado está de acordo com alguns estudos sobre a descoloração de vários corantes utilizando algas e cianobactérias, que constataram que mais de $70 \%$ dos corantes foram removidos quase instantaneamente. Este resultado sugere que a remoção relativamente rápida pode ser principalmente devido à adsorção dos corantes na superfície celular dos biossorventes ${ }^{25,26,27}$.

Abiossorçãoemcianobactériaséatribuídaprincipalmente às propriedades da sua parede celular formada por uma rede macromolecular tridimensional porosa. Esta possui componentes importantes, como: peptideoglicano, ácido teicóico, alginato, polissacarídeos e proteínas, e apresenta também grupos carboxílicos, hidroxílicos e fosfatos. Esses grupos são polieletrólitos carregados que se acredita serem responsáveis pelo sequestro de materiais indesejáveis dos efluentes industriais, permitindo que a biossorção ocorra na parede celular ${ }^{28-31}$.

A camada de peptideoglicano das cianobactérias desempenha um papel importante na ligação de metais, pois é consideravelmente espessa, o que mostra um maior grau de reticulação entre as cadeias polissacarídicas em comparação com bactérias Gram-negativas ${ }^{6,32}$. Além disso, esta possui uma grande área superficial com sítios ativos capazes de proverem ligações rápidas e reversíveis de cátions, sendo um mosaico de sítios trocadores catiônicos e aniônicos ${ }^{33}$.

A adsorção passiva em algas e cianobactérias é um exemplo de biossorção, sendo um processo de ligação passiva de cátions pela biomassa seca ou viva ${ }^{30}$. Esta adsorção é explicada pela ligação passiva dos cátions a parede celular das algas, $m$ que a atração eletrostática e a complexação podem desempenhar um papel na adsorção dos corantes ${ }^{31,34}$.

\section{Conclusão}

$\mathrm{Na}$ avaliação da capacidade de remoção da cor do corante Acid Blue 324, adicionado ao meio de cultura com a presença da Spirulina maxima, alcançou-se uma remoção máxima da cor de 83,19\% em 84 minutos de ensaio, valor este muito próximo aos valores máximos de captação encontrados na literatura para outras biomassas e sorbatos.

Com o planejamento experimental pode-se visualizar quais variáveis de entrada deveriam ser escolhidas para apresentar um maior percentual de remoção do corante. Tal planejamento mostrou que para obtermos maiores valores de remoção, é preciso trabalhar com as maiores concentrações de corante e do nitrato, também mostrou que o nitrato não foi uma boa escolha de variável de entrada, pois teve pouca influência no experimento.

É possível relatar que a cianobactéria Spirulina maxima apresenta bons resultados na remoção da cor, inserindo o corante no meio de cultura, observou-se uma redução expressiva da cor produzindo uma solução clarificada.

\section{Referências}

1. Rossi, T. R.; Tanaka, Y. H.; Cano, V.; Andrade, H; Miyadahira, C. A. G; Nolasco, M.; Costa, S. M.; Costa, S. A. Caracterização de Efluente Têxtil Tratado por Adsorção com Adsorvente Residual Gerado na Indústria de Alumínio. 6th International Workshop, Advances in Cleaner Production. São Paulo. Brazil, 2017.

2. Kolekar, Yogesh M.; Kodam, Kisan M. Decolorization of textile dyes by Alishewanella sp. KMK6. Appl Microbiol Biotechnol., 2012, 95, 521-529.

3. Mounteer, A. H.; Mokfienskim, J. J.; Amorim, F. R. Remoção de matéria orgânica recalcitrante de efluentes de celulose kraft de branqueamento por ozonólise. Congresso Anual de Celulose e Papel. São Paulo, 2004.

4. Daneshvar, N.; Ayazloo, M.; Khataee A. R.; Pourhassan, M. Biological decolorization of dye solution containing Malachite Green by microalgae Cosmarium sp. Bioresource Technology, 2007, 98, 1176-1182.

5. Hitz, H. R.; Huger, W.; Reed, R. H.; SOC, J. Dyes and Colour, 1978.

6. De Philippis, R.; Colica, G.; Micheletti, E. Exopolysaccharideproducing cyanobacteria in heavy metal removal from water: molecular basis and practical applicability of the biosorption process. Appl Microbiol Biotechnol. 2011, 92, 697-708.

7. Sulaymon, A. H.; Mohammed, A. A.; Al-Musawi, T. J. Competitive biosorption of lead, cadmium, copper, and arsenic ions using algae. Environ Sci Pollut Res. 2013, 20, 3011-3023.

8. Derner, R. B. Microalgas, produtos e aplicações. Ciência Rural, 2006, 36, 6, 1959-1967.

9. Huang, G.; Chen, F.; Wei, D.; Zhang, X.; Chen, G. Biodiesel production by microalgal biotechnology. Appl. Energ., 2010, 87, 38-46.

10. Picardo, M. C. Desempenho de Isochrysis galbana na Produção 
de Óleo e Sequestro de CO2 com Fotobiorreator Piloto. Tese de Doutorado. Universidade Federal do Rio de Janeiro - UFRJ, Programa de Pós Graduação em Tecnologia de Processos Químicos e Bioquímicos, 2012.

11. Afreen, S.; Bano, F.; Ahmad, N.; Fatma, T. Screeningand optimization of laccase from cyanobacteria with its potential in decolorization of anthraquinonic dye Remazol Brilliant Blue R. Biocatalysis and Agricultural Biotechnology, Department of Biology, Taibah University, Medina, Saudi Arabia, 2017.

12. Montgomery, D.; Calado, V. Planejamento de Experimentos usando o Statistica. [s.1.]: E-papers Serviços Editoriais Ltda, 2003, 260.

13. Teixeira, Y. F. Avaliação do uso de microalgas como possível alternative para a remoção de cor de corantes têxteis. Monografia do curso de Bacharelado em Engenharia Química. Faculdade SENAICETIQT. Rio de Janeiro, 2016.

14. Aiba, S.; Ogawa, T. Assessment of Growth Yield of a Blue-green Alga, Spirulina platensis, in Axenic and Continuous Culture. Journal of General Microb., 1977, 102, 179-182.

15. Ozer, A.; Akkayaa, G.; Turabik, M. The biosorption of Acid Red 337 and Acid Blue 324 on Enteromorpha prolifera: The application of nonlinear regression analysis to dye biosorption. Chemical Engineering Journal, 2005, 112, 181-190.

16. Ozer, A.; Akkayaa, G.; Turabik, M. Biosorption of Acid Blue 290 (AB 290) and Acid Blue 324 (AB 324) dyes on Spirogyra rhizopus, Journal of Hazardous Materials, 2006, 135, 355-364.

17. Collos Y.; Mornet F.; Sciandra A.; Waser N.; Larson A.; Harrison P. J. The optical method for the rapid measurement of micromolar concentrations of nitrate in marine phytoplankton cultures. Journal of App. Phycology. 1999, 11, 179-184.

18. Zhu, C. J.; Lee, Y. K. Determination of biomass dry weight of marine microalgae. Journal of Appl. Phycology. 1997, 9, 189-194.

19. Nishikawa, E. Bioadsorção de Cádmio em Resíduo da Extração de Alginato da Alga Marinha Sargassum Filipendula. Dissertação de Mestrado. Faculdade de Engenharia Química. Universidade Estadual de Campinas, SP. 2017.

20. Pedro, A. S.; González-lópez, C.V.; Acién, F.G.; Molina-grima, E. "Marine microalgaeselectionandcultureconditionsoptimization for biodiesel production". Bioresource Technology, 2013, 134, 353-361.

21. Nelson, D. L.; Cox, M. M. Princípios de Bioquímica, Sarvier, São Paulo, Brasil, ed. 4th, 2004.

22. Lourenço, S. O. Cultivo de microalgas marinhas: princípios e aplicações. São Carlos: Rima, 2006.

23. Das, P.; Obbard, J. P. Incremental energy supply for microalgae culture in a photobioreactor. Bioresource Technology, 2011, 102, 2973-2978.

24. Subhash, G. V.; Rohit, M.V.; Devi, M. P.; Swamy, Y.V.; Mohan, S. $\mathrm{V}$. Temperature induced stress influence on biodiesel productivity during mixotrophic microalgae cultivation with wastewater. Bioresour. Technol, 2014.

25. Chen, K.; Wu, J.; Liou, D., Sz-Chwun, J. Decolorization of the textile dyes by newly isolated bacterial strains. Journal of Biotechnology, 2003, 101, 57-68.

26. Shah, V.; Garg, N.; Madamwar, D. An integrated process of textile dye removal and hydrogen evolution using cyanobacterium, Phormidium valderianum. World Journal of Microb. \& Biotechn., 2001, 17, 499-504.

27. Daneshvar, N.; Ayazloo, M.; Khataee A. R.; Pourhassan, M. Biological decolorization of dye solution containing Malachite Green by microalgae Cosmarium sp. Bioresource Technology, 2007, 98, 1176-1182.

28. Vieira, R. H. S. F.; Volesky, B. Biosorption: a solution to pollution?. INTERNATL MICROBIOL, 2000, 3, 17-24.

29. Davis, T. A.; Volesky, B.; Mucci, A.; A review of the biochemistry of heavy metal biosorption by brown algae. Water Research, 2003, 37, 4311-4330.

30. Chojnacka, K.; Chojnacki, A.; Gorecka, H. Biosorption of Cr3+, $\mathrm{Cd} 2+$ and $\mathrm{Cu} 2+$ ions by blue-green algae Spirulina sp.: kinetics, equilibrium and the mechanism of the process. Chemosphere, 2005, $59,75-84$

31. Ozer, A.; Akkayaa, G.; Turabik, M. Biosorption of Acid Blue 290 (AB 290) and Acid Blue 324 (AB 324) dyes on Spirogyra rhizopus. Journal of Hazardous Materials, 2006, 135, 355-364.

32. Sharma, N. K.; Tiwari, S. P.; Tripathi, K.; Rai, A. K. Sustainability and cyanobacteria (blue-green algae): facts and challenges. J Appl Phycol, 2011, 23, 1059-1081.

33. Silva, D. M. S. Estudo do processo adsortivo de cádmio por algas Arribadas. Dissertação de Mestrado. Universidade Federal de Pernambuco. Programa de Pós-Graduação em Engenharia Química. Recife, 2010.

34. Aneja, Rajiv Kumar; Chaudhary, Gunjan; Ahluwalia, Sarabjeet Singh; Goyal, Dinesh. "Biosorption of $\mathrm{Pb} 2+$ and $\mathrm{Zn} 2+$ by NonLiving Biomass of Spirulina sp." Indian J Microbiol, 2010, 50, 4, 438-442.

\section{Vanessa T. Pereira, Ronaldo L. de Souza, Flávia D. Pimenta \& Marta C. Picardo*}

Centro de Tecnologia da Indústria Química e Têxtil (Faculdade SENAI CETIQT), Rua Magalhães Castro, 174, Riachuelo, CEP: 20961-020 - Rio de Janeiro, RJ, Brasil.

*E-mail: mcpicardo@cetiqt.senai.br 